\title{
Double Electron Spin Resonance of Engineered Atomic Structures on a Surface
}

\section{Soo-Hyon Phark}

Institute for Basic Science

Yi Chen

Institute for Basic Science

Christoph Wolf

Institute for Basic Science

Hong Bui

Ewha Womans University

\section{Yu Wang}

Institute for Basic Science

Masahiro Haze

University of Tokyo

Jinkyung Kim

Ewha Womans University

Christopher Lutz

IBM Research - Almaden https://orcid.org/0000-0002-7993-4759

Andreas Heinrich ( $\square$ heinrich.andreas@qns.science)

Institute for Basic Science at Ewha Womans University https://orcid.org/0000-0001-6204-471X

Yujeong Bae

IBM Almaden Research Center https://orcid.org/0000-0002-9983-8529

\section{Article}

Keywords: quantum devices, scanning tunneling microscopes

Posted Date: September 8th, 2021

DOI: https://doi.org/10.21203/rs.3.rs-871457/v1

License: (9) This work is licensed under a Creative Commons Attribution 4.0 International License.

Read Full License 


\title{
Title: Double Electron Spin Resonance of Engineered Atomic Structures on a
}

\section{Surface}

\section{Authors:}

Soo-Hyon Phark ${ }^{1,2,3 \dagger}$, Yi Chen ${ }^{1,3 \dagger}$, Christoph Wolf ${ }^{1,3}$, Hong T. Bui ${ }^{1,4}$, Yu Wang ${ }^{1,3}$, Masahiro Haze $^{1,5}$, Jinkyung Kim ${ }^{1,4}$, Christopher P. Lutz ${ }^{2 *}$, Andreas J. Heinrich ${ }^{1,4^{*}}$, Yujeong Bae ${ }^{1,4^{*}}$

\section{Affiliations:}

${ }^{1}$ Center for Quantum Nanoscience, Institute for Basic Science (IBS), Seoul 03760, Korea

${ }^{2}$ IBM Research Division, Almaden Research Center, San Jose, CA 95120, USA.

${ }^{3}$ Ewha Womans University, Seoul 03760, Korea

${ }^{4}$ Department of Physics, Ewha Womans University, Seoul 03760, Korea

${ }^{5}$ The Institute for Solid State Physics, University of Tokyo, Kashiwa 277-8581, Japan

*Corresponding author. Email: cplutz@us.ibm.com, heinrich.andreas@qnns.science, bae.yujeong@quns.science

$\uparrow$ These authors contributed equally to this work.

\begin{abstract}
Atomic-scale control of multiple spins with individual addressability enables the bottom-up design of functional quantum devices. Tailored nanostructures can be built with atomic precision using scanning tunneling microscopes, but quantum-coherent driving has thus far been limited to a spin in the tunnel junction. Here we show the ability to drive and detect the spin resonance of a remote spin using the electric field from the tip and a single-atom magnet placed nearby. Read-out was achieved via a weakly coupled second spin in the tunnel junction that acted as a quantum sensor. We simultaneously and independently drove the sensor and remote spins by two radio frequency voltages in double resonance experiments, which provides a path to quantumcoherent multi-spin manipulation in customized spin structures on surfaces.
\end{abstract}


One-Sentence Summary: Using a scanning tunneling microscope, we simultaneously control two spins using one tip, paving the way for multi-spin-qubit operations on surfaces.

\section{Main Text:}

Fabricating and controlling functional quantum devices with atomic precision is one of the long-term goals of quantum-coherent nanoscience (1). Quantum-coherent control of individual spins has been achieved in nanoscale systems, including dopants in semiconductors (2), color centers in insulators (3), semiconductor quantum dots (4), and molecular devices (5). However, building quantum systems with atomic scale control of their architecture and the microscopic interactions therein presents a major challenge (6). Individual spins, localized in atoms or molecules on surfaces, are a new candidate for realizing qubits, where atomically-precise fabrication can be achieved using a scanning tunneling microscope (STM). Quantum control of single atoms on surfaces driven by a magnetic tip has been demonstrated through continuous-wave (7-11) and pulsed (12) electron spin resonance (ESR) in STM. The STM can readily assemble multi-spin quantum systems $(13,14)$ but use of ESR to coherently control a spin that is remote from the tunnel junction has not yet been considered (15-18).

Here we demonstrate the ability to drive the resonance and sense the resulting spin state of a remote spin that is not directly in the tunnel junction. The key ingredient for the remote driving is a single-atom magnet placed in atomic proximity to the remote spin whose spatially inhomogeneous magnetic field converts the applied radio-frequency (RF) electric field into a driving magnetic field. To detect the quantum state of the remote spin, we positioned a nearby spin-resonant atom as a quantum sensor in the tunnel junction and performed double resonance experiments. 
Experiments were performed in an ultra-high vacuum STM systems operating at $0.4 \mathrm{~K}$ (19). Two atomic spins were positioned on a surface so that they weakly coupled to each other with energy $J_{1,2}$ (Figs. 1A and B). Both spins were a spin-1/2 hydrogenated Ti atom on bilayer $\mathrm{MgO} / \mathrm{Ag}$ substrate $(10,20,21)$, hereafter referred to as Ti-1 or "sensor spin", and Ti-2 or "remote spin". An Fe atom was placed in close proximity to Ti-2 to give a coupling strength of $J_{2, \mathrm{Fe}}$. The Fe atom's spin $(S=2)$ remained aligned perpendicular to the $\mathrm{MgO}$ surface due to its large easyaxis magnetic anisotropy and flipped up and down infrequently compared to the Rabi times used here $(7,22)$. The resonance of the sensor spin was driven by the inhomogeneous magnetic field generated from the coupling with the tip $(16,18)$, whereas the remote spin was driven by its interaction with the nearby Fe. Ti-1 Ti-2

To characterize the interactions in the three-atom structure, we first acquired ESR-STM spectra of the Ti spins in the structure by applying a single-frequency RF voltage to the tunnel junction (Fig. 1C; figs. S1 and S2). The spectrum of Ti-1 (red curve in Fig. 1C) shows two ESR peaks that are separated by the coupling between Ti- 1 and Ti-2 of $J_{1,2}=0.112 \mathrm{GHz}$, in good agreement with previous studies $(20,21)$. Coupling between Ti-2 and Fe created additional splitting, as evidenced by the four ESR peaks measured with the tip positioned on Ti-2 (blue curve in Fig. 1C), resulting in $J_{2, \mathrm{Fe}}=3.891 \mathrm{GHz}$ between Ti-2 and Fe (fig. S2). Coupling of Ti-1 to the Fe spin was negligible. ESR transitions of Fe were far from the frequency range used here (7) and are neglected in the following. The presence of the Fe atom resulted in significantly different Zeeman energies for Ti-1 and Ti-2, so their spin eigenstates are well-described as Zeeman product states $\left|S_{z}(\mathrm{Ti}-1) S_{z}(\mathrm{Ti}-2)\right\rangle:|00\rangle,|01\rangle,|10\rangle,|11\rangle$, as depicted in Fig. 1C. In this work we focus on the four ESR resonances $\left(f_{0 \uparrow}, f_{1 \uparrow}, f_{\uparrow 0}, f_{\uparrow 1}\right)$, where 0,1 , and $\uparrow$ indicate that the corresponding spin is in the ground, excited, and resonant states, respectively. 
To demonstrate the remote driving of Ti-2, we performed double-resonance ESR measurements by positioning the tip above Ti-1 and monitoring one of its two resonances while sweeping a second RF frequency across the Ti-2 resonances (Fig. 2 and fig. S3C). This scheme is a nanoscale implementation of the double-resonance technique used in conventional ensemble $\operatorname{ESR}(23,24)$. At $f_{\mathrm{RF} 1}=f_{\mathfrak{}}$, the spectra showed two peaks, separated by the coupling energy $J_{1,2}$ (Fig. 2A). Note that a single-frequency ESR signal is negative in our measurements (Fig. 1C), therefore peaks in a double-resonance spectrum correspond to a reduction of the ESR signal of Ti-1. This reduction in the Ti-1 ESR signal stems from a transfer of state population from $|00\rangle$ to $|01\rangle$ due to resonant driving of the remote spin, Ti-2 (19). For comparison, the spectra obtained at $f_{\mathrm{RF} 1}=f_{\uparrow 1}$ (Fig. 2B) showed resonances at the same frequencies, but they appear as dips instead of peaks because of an increase in the ESR signal of Ti-1. We found that the resonance frequencies in these double-resonance spectra were $\sim 400 \mathrm{MHz}$ higher than those in the single-frequency ESR with the tip positioned on Ti-2 (Fig. 1C), indicating that the tip's magnetic field (fig. S1) is absent when the Ti-2 spin is being remotely driven.

To show the origin of the double-resonance contrast more directly, we acquired a series of ESR spectra of Ti-1 with increasing $V_{\mathrm{RF} 2}$ at one of the Ti-2 resonances (Fig. 1D). For $V_{\mathrm{RF} 2}=0$, this experiment is identical to single-frequency ESR of Ti-1 (Fig. 1C), where a larger peak height is seen at $f_{\uparrow 0}\left(I_{\uparrow 0}\right)$ than at $f_{\uparrow 1}\left(I_{\uparrow 1}\right)$, with the ratio $I_{\uparrow 0} / I_{\uparrow 1}$ given by the thermal population of the Ti-2 spin states. With increasing $V_{\mathrm{RF} 2}$, we observed a pronounced, simultaneous decrease of $I_{\mathfrak{}}$ and increase of $I_{\uparrow 1}$ (Fig. 1D and fig. S4). Therefore, the double-resonance peaks and dips observed in Fig. 2 can be understood as the change of the ESR peak height with $V_{\mathrm{RF} 2}$ in Fig. 1D (figs. S3 and S4). 
When $V_{\mathrm{RF} 1}$ was increased beyond the level used in Fig. 2, a splitting of the doubleresonance peaks was observed, whose spacing increased linearly with $V_{\mathrm{RF} 1}$ (Fig. 3). This is a manifestation of the AC Stark effect (25), where the states $|00\rangle$ and $|10\rangle$ were split into two doublets by the strong interaction between the Ti-1 spin and its resonant RF field $(19,26,27)$. The splitting of the $|00\rangle$ state was spectroscopically probed by the $f_{0 \uparrow}$ transition (Fig. 3 inset). The splitting in the doublets directly corresponds to the Rabi rate $\Omega^{(1)}$ of Ti-1, which we found to be proportional to $V_{\mathrm{RF} 1}$, allowing us to measure the Rabi rate of Ti-1, $\Omega^{(1)} /\left(2 \pi V_{\mathrm{RF} 1}\right)=0.160 \pm$ $0.015 \mathrm{MHz} / \mathrm{mV}$ (Fig. 3B). By reversing the roles of the two RF voltages, the AC Stark splitting was also observed when sweeping $f_{\mathrm{RF} 1}$ (Fig. 1D and fig. S8), giving the remote driving Rabi rate of Ti-2, which reveals $\Omega^{(2)} /\left(2 \pi V_{\mathrm{RF} 2}\right)=0.250 \pm 0.013 \mathrm{MHz} / \mathrm{mV}$. This showcases that the strength of the remote driving is similar to that of the local driving by the tip.

We conducted several control experiments to show the data reproducibility and to confirm that the remote driving of Ti-2 is due to the presence of the nearby Fe atom. First, we repeated the double-resonance measurements using another STM system on a Ti-Ti-Fe structure of different spacing between Ti-2 and Fe (figs. S9-S11). Second, we observed that the double-resonance frequencies of Ti-2 were independent of the tip position on Ti-1 (unlike Ti-1 single ESR spectra), showing the negligible effects of the tip's magnetic field on the remote spin (fig. S12). Third, we positioned the tip on Ti-2 and attempted to remotely drive Ti-1, which showed no ESR signal presumably due to the weakness of the interaction with $\mathrm{Fe}$ (fig. S13).

We performed simulations of an open two-spin quantum system (28) based on the Lindblad formalism (29), which reproduced all double resonance spectra (Figs. 1D and 2) (19). Using the Rabi rates and resonance frequencies determined from the experiments, we derived energy relaxation times for the Ti-1 spin, giving $T_{1}^{(1)}=8 \pm 1 \mathrm{~ns}$ from fits to the linewidth in the double 
resonance data (fig. S14). We found a much longer lifetime for Ti-2, the remote spin, obtaining $T_{1}^{(2)}=150 \pm 10 \mathrm{~ns}$ by fitting the double-resonance spectra (fig. S14) and the peak height ratio

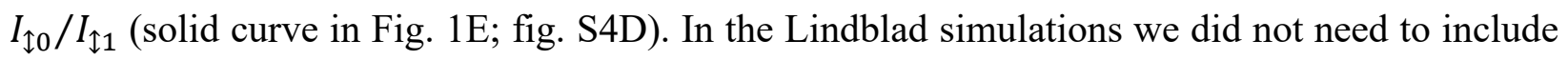
a pure dephasing channel, indicating that $T_{1}$ processes were the dominant source of dephasing (fig. S15). We note that the average interval between successive tunneling electrons at a tunnel current of $20 \mathrm{pA}$ is $8 \mathrm{~ns}$, suggesting the dominant role of tunneling electrons in the energy relaxation process of the sensor spin, Ti-1 (10). On the other hand, the large relaxation time of Ti-2 strongly indicates the absence of tunnel current-induced relaxation for the remote spin. In addition, an easily accessible $\mathrm{RF}$ voltage, $V_{\mathrm{RF} 2} \approx 40 \mathrm{mV}$ (Fig. 1E), was enough to remotely drive the populations of Ti-2 states to saturation (Fig. 1E). An intuitive understanding of these population transfers can be developed using a rate equation approach (19).

To further investigate the underlying mechanism of the remote driving, we formed an isolated Ti-Fe pair and performed single-frequency ESR spectroscopy on Ti (Fig. 4A). We measured ESR spectra on Ti as a function of tip-sample distance and found two resonance peaks, corresponding to the two Fe spin states $|\Uparrow\rangle$ and $|\Downarrow\rangle$, respectively. We observed a strongly nonmonotonic change of the height for one of the peaks, which vanished at an intermediate tip-atom distance (Fig. 4, figs. S18 and S19). This non-monotonic behavior was not observed for isolated Ti (11) (fig. S1) and strongly implies the presence of two competing transverse RF driving fields, one induced by the tip $\left(\boldsymbol{B}_{\mathrm{RF}, \mathrm{tip}}^{(\perp)}\right)$ and the other by $\mathrm{Fe}\left(\boldsymbol{B}_{\mathrm{RF}, \mathrm{Fe}}^{(\perp)}\right)$. For this particular tip, the two driving fields were parallel, adding to each other for the Fe in the $|\Uparrow\rangle$ state, and canceling each other for Fe $|\Downarrow\rangle$ (Fig. 4C insets; fig. S18). This specific behavior was observed only with selective tips (fig. S19) and not for the tip used in Figs. 1-3. Other tips showed a similar behavior but generally 
yielded incomplete cancellation (fig. S20). The finite ESR peak heights when the tip is far away from the atom demonstrates a sizable $\boldsymbol{B}_{\mathrm{RF}, \mathrm{Fe}}^{(\perp)}$ at the Ti position by the nearby Fe.

The remote spin driving can be understood by the piezoelectric driving mechanism of ESRSTM. The tip-supplied RF electric field acts on an extended area with a radius of a few nanometers across the surface (Fig. 1A) (30) and induces an adiabatic piezoelectric motion (16). This motion, in the presence of an inhomogeneous magnetic field that originates either from a magnetic tip or from a single-atom magnet on the surface, results in an oscillating transverse magnetic field that drives the ESR transitions. Other single-atom magnets with larger magnetic moments and longer spin relaxation times, such as Ho (31) and Dy (32), might provide stronger remote driving than Fe.

Our results demonstrate the ability to drive the resonance of spins that are not in the tunnel junction. These experiments, combined with simulations using an open quantum spin model, reveal that the remote spin, free from the tunnel current, has a 10-fold longer relaxation time than the sensor spin while retaining a comparable Rabi rate. Our discovery enables coherent manipulation of coupled multiple spins using one STM tip. Quantum sensing (33) and simulation (34) using multiple spins on a surface are now within reach. 


\section{References and Notes}

1. A. J. Heinrich, W. D. Oliver, L. Vandersypen, A. Ardavan, R. Sessoli, D. Loss, A. B. Jayich, J. Fernandez-Rossier, A. Laucht, A. Morello, Quantum-coherent Nanoscience. Nature Nanotechnology, accepted (2021).

2. J. J. Pla, K. Y. Tan, J. P. Dehollain, W. H. Lim, J. J. L. Morton, D. N. Jamieson, A. S. Dzurak, A. Morello, A single-atom electron spin qubit in silicon. Nature 489, 541-545 (2012).

3. L. Childress, M. V. Gurudev Dutt, J. M. Taylor, A. S. Zibrov, F. Jelezko, J. Wrachtrup, P. R. Hemmer, M. D. Lukin, Coherent Dynamics of Coupled Electron and Nuclear Spin Qubits in Diamond. Science 314, 281-285 (2006).

4. L. M. K. Vandersypen, M. A. Eriksson, Quantum computing with semiconductor spins. Physics Today 72, 38-45 (2019).

5. S. Thiele, F. Balestro, R. Ballou, S. Klyatskaya, M. Ruben, W. Wernsdorfer, Electrically driven nuclear spin resonance in single-molecule magnets. Science 344, 1135-1138 (2014).

6. Y. He, S. K. Gorman, D. Keith, L. Kranz, J. G. Keizer, M. Y. Simmons, A two-qubit gate between phosphorus donor electrons in silicon. Nature 571, 371-375 (2019).

7. S. Baumann, W. Paul, T. Choi, C. P. Lutz, A. Ardavan, A. J. Heinrich, Electron paramagnetic resonance of individual atoms on a surface. Science 350, 417-420 (2015).

8. T. S. Seifert, S. Kovarik, D. M. Juraschek, N. A. Spaldin, P. Gambardella, S. Stepanow, Longitudinal and transverse electron paramagnetic resonance in a scanning tunneling microscope. Science Advances 6, eabc5511 (2020).

9. M. Steinbrecher, W. M. J. van Weerdenburg, E. F. Walraven, N. P. E. van Mullekom, J. W. Gerritsen, F. D. Natterer, D. I. Badrtdinov, A. N. Rudenko, V. V. Mazurenko, V. Vladimir, M. I. Katsnelson, A. van der Avoird, G. C. Groenenboom, A. Khajetoorians, Quantifying the interplay between fine structure and geometry of an individual molecule on a surface. Physical Review B 103, 155405 (2021).

10. L. M. Veldman, L. Farinacci, R. Rejali, R. Broekhoven, J. Gobeil, D. Coffey, M. Ternes, A. F. Otte, Free coherent evolution of a coupled atomic spin system initialized by electron scattering. Science 372, 964-968 (2021).

11. J. K. Kim, W. Jang, H. T. Bui, D. Choi, C. Wolf, F. Delgado, D. Krylov, S. Lee, S. Yoon, C. P. Lutz, A. J. Heinrich, Y. Bae, Spin Resonance Amplitude and Frequency of a Single Atom on a Surface in a Vector Magnetic Field. arXiv:2103.09582 (2021).

12. K. Yang, W. Paul, S. H. Phark, P. Willke, Y. Bae, T. Choi, T. Esat, A. Ardavan, A. J. Heinrich, C. P. Lutz, Coherent spin manipulation of individual atoms on a surface. Science 366, 509-512 (2019).

13. A. A. Khajetoorians, D. Wegner, A. F. Otte, I. Swart, Creating designer quantum states of matter atom-by-atom. Nature Reviews Physics 1, 703-715 (2019).

14. K. Yang, S. H. Phark, Y. Bae, T. Esat, P. Willke, A. Ardavan, A. J. Heinrich, C. P. Lutz, Probing resonating valence bond states in artificial quantum magnets. Nature Communications 12, 993 (2021).

15. P. Berggren, J. Fransson, Electron Paramagnetic Resonance of Single Magnetic Moment on a Surface. Scientific Reports 6, 25584 (2016).

16. J. L. Lado, A. Ferrón, J. Fernández-Rossier, Exchange mechanism for electron paramagnetic resonance of individual adatoms. Physical Review B 96, 205420 (2017). 
17. A. M. Shakirov, A. N. Rubtsov, P. Ribeiro, Spin transfer torque induced paramagnetic resonance. Physical Review B 99, 054434 (2019).

18. J. Reina Gálvez, C. Wolf, F. Delgado, N. Lorente, Cotunneling mechanism for allelectrical electron spin resonance of single adsorbed atoms. Physical Review B 100, 035411 (2019).

19. See Supplementary Materials.

20. K. Yang, Y. Bae, W. Paul, F. D. Natterer, P. Willke, J. L. Lado, A. Ferrón, T. Choi, J. Fernández-Rossier, A. J. Heinrich, C. P. Lutz, Engineering the Eigenstates of Coupled Spin-1/2 Atoms on a Surface. Physical Review Letters 119, 227206 (2017).

21. Y. Bae, K. Yang, P. Willke, T. Choi, A. J. Heinrich, C. P. Lutz, Enhanced quantum coherence in exchange coupled spins via singlet-triplet transitions. Science Advances $\mathbf{4}$, eaau4159 (2018).

22. W. Paul, K. Yang, S. Baumann, N. Romming, T. Choi, C. P. Lutz, A. J. Heinrich, Control of the millisecond spin lifetime of an electrically probed atom. Nature Physics 13, 403407 (2017).

23. G. Feher, Electron Spin Resonance Experiments on Donors in Silicon. I. Electronic Structure of Donors by the Electron Nuclear Double Resonance Technique. Physical Review 114, 1219-1244 (1959).

24. L. D. K. Larry Kevan, Electron spin double resonance spectroscopy. (Wiley, New York, 1976).

25. S. H. Autler, C. H. Townes, Stark Effect in Rapidly Varying Fields. Physical Review 100, 703-722 (1955).

26. A. Laucht, R. Kalra, S. Simmons, J. P. Dehollain, J. T. Muhonen, F. A. Mohiyaddin, S. Freer, F. E. Hudson, K. M. Itoh, D. N. Jamieson, J. C. McCallum, A. S. Dzurak, A. Morello, A dressed spin qubit in silicon. Nature Nanotechnology 12, 61-66 (2017).

27. K. C. Miao, J. P. Blanton, C. P. Anderson, A. Bourassa, A. L. Crook, G. Wolfowicz, H. Abe, T. Ohshima, D. D. Awschalom, Universal coherence protection in a solid-state spin qubit. Science 369, 1493-1497 (2020).

28. P. D. N. J. R. Johansson, F. Nori, QuTiP 2: A Python framework for the dynamics of open quantum systems. Comp. Phys. Comm. 184, 1234 (2013).

29. D. Manzano, A short introduction to the Lindblad master equation. AIP Advances 10, 025106 (2020).

30. F. Krok, K. Sajewicz, J. Konior, M. Goryl, P. Piatkowski, M. Szymonski, Lateral resolution and potential sensitivity in Kelvin probe force microscopy: Towards understanding of the sub-nanometer resolution. Physical Review B 77, 235427 (2008).

31. F. Donati, S. Rusponi, S. Stepanow, C. Wäckerlin, A. Singha, L. Persichetti, R. Baltic, K. Diller, F. Patthey, E. Fernandes, J. Dreiser, Ž. Šljivančanin, K. Kummer, C. Nistor, P. Gambardella, H. Brune, Magnetic remanence in single atoms. Science 352, 318-321 (2016).

32. A. Singha, P. Willke, T. Bilgeri, X. Zhang, H. Brune, F. Donati, A. J. Heinrich, T. Choi, Engineering atomic-scale magnetic fields by dysprosium single atom magnets. Nature Communications 12, 4179 (2021).

33. C. L. Degen, F. Reinhard, P. Cappellaro, Quantum sensing. Reviews of Modern Physics 89, 035002 (2017).

34. A. Trabesinger, Quantum simulation. Nature Physics 8, 263-263 (2012). 
35. W. Paul, S. Baumann, C. P. Lutz, A. J. Heinrich, Generation of constant-amplitude radiofrequency sweeps at a tunnel junction for spin resonance STM. Review of Scientific Instruments 87, 074703 (2016).

36. J. C. R. Chiao, Quantum Optics. (Oxford University Press, Oxford, 2014). 
Acknowledgments: We thank A. Ardavan, M. Ternes, and J. F. Rossier for fruitful discussions.

Funding: This work was supported by the Institute for Basic Science (IBS-R027-D1). CPL acknowledges support from the Office of Naval Research, grant N00014-21-1-2467. MH acknowledges financial support from University of Tokyo Global Activity Support Program for Young Researchers (FY2020).

Author contributions: CPL, SP, and AJH conceived the experiment. YC and YB designed the measurement schemes. SP, YC, HTB, YW, MH, JK, and YB performed the experiments. $\mathrm{YC}, \mathrm{CW}$, and $\mathrm{AJH}$ carried out theoretical calculations. All authors contributed to data analysis and manuscript preparation.

Competing interests: The authors declare no competing interests.

Data and materials availability: The data that support the findings of this study are available from the corresponding authors upon reasonable request.

\section{Supplementary Materials}

Materials and Methods

Supplementary Text

Figs. S1 to S20 

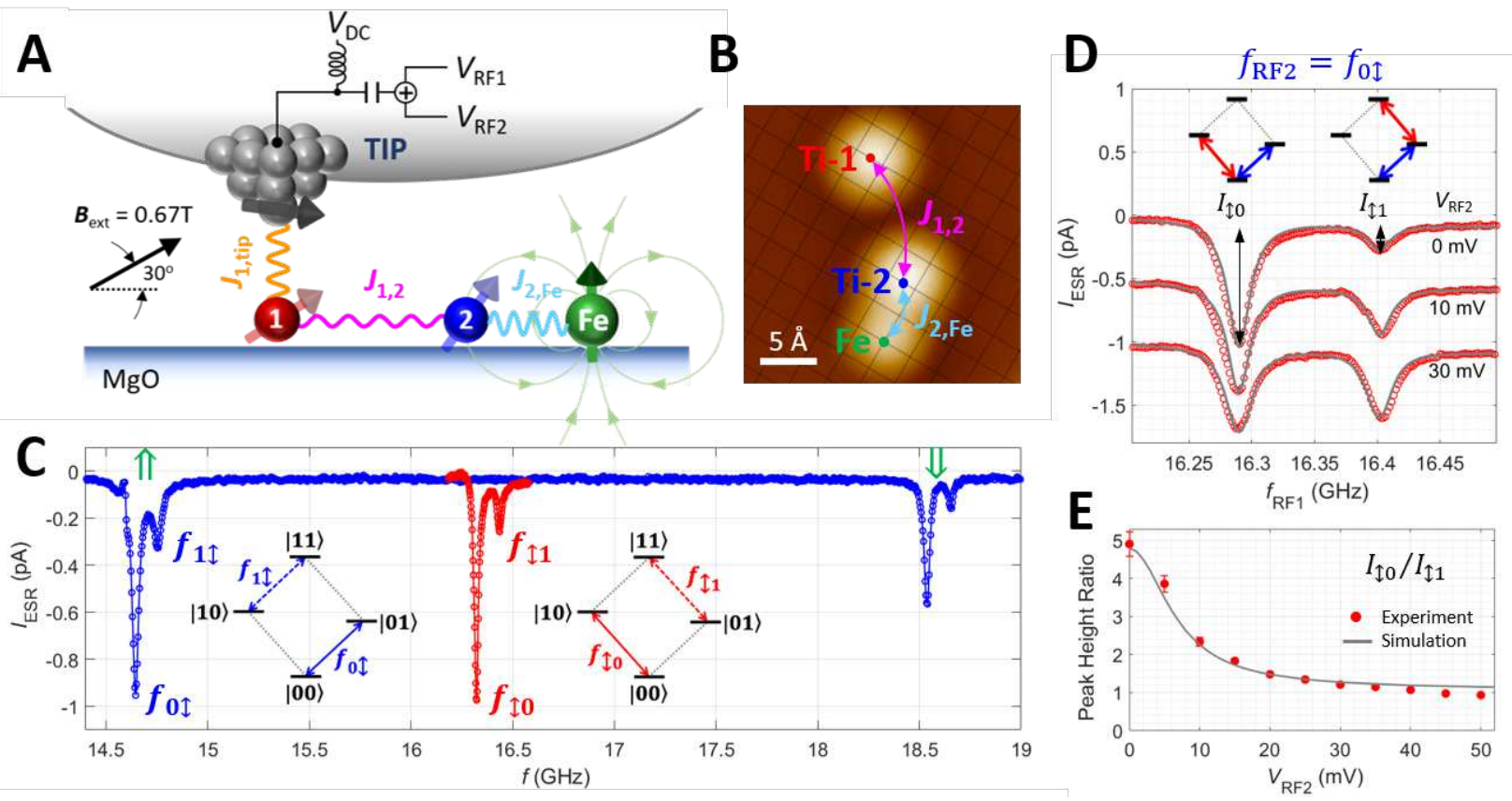

Fig. 1. Engineered atomic structures for double resonance experiments. (A) Schematic of double resonance spectroscopy of two coupled Ti spins on a surface using an ESR-STM integrated with two RF sources, $V_{\mathrm{RF} 1}$ and $V_{\mathrm{RF} 2}$. (B) STM topographic image of an engineered nanostructure composed of sensor spin (Ti-1), remote spin (Ti-2), and Fe adatom. The Ti-1 to Ti-2 spacing is $1.22 \mathrm{~nm}$, and Ti-2 to Fe is $0.59 \mathrm{~nm}$. The black grid indicates the oxygen sites of the underlying $\mathrm{MgO}$ lattice $\left(I_{\mathrm{DC}}=10 \mathrm{pA}, V_{\mathrm{DC}}=100 \mathrm{mV}\right)$. (C) Single-frequency ESR spectra measured on Ti-1 (red) and Ti-2 (blue). Inset: energy diagrams of two weakly coupled spins with four spin states labelled according to $\mid S_{Z}$ (Ti-1), $S_{Z}$ (Ti-2) $\rangle$ and ESR transitions corresponding to two peaks for each spin. $\Uparrow$ and $\Downarrow$ indicate the Fe spin states. (D) Dual-frequency ESR driving of coupled two-spin system. Ti-1 ESR spectra with an increasing $V_{\mathrm{RF} 2}$, with $f_{\mathrm{RF} 2}$ fixed at a Ti-2 resonance $f_{0 \uparrow}=$ $15.071 \mathrm{GHz}$. Successive spectra are shifted by $-0.5 \mathrm{pA}\left(I_{\mathrm{DC}}=20 \mathrm{pA}, V_{\mathrm{DC}}=50 \mathrm{mV}, V_{\mathrm{RF} 1}=\right.$ $30 \mathrm{mV}, 0.4<T<0.5 \mathrm{~K}$. (E) $V_{\mathrm{RF} 2}$-dependence of peak height ratio $I_{\uparrow 0} / I_{\uparrow 1}$ extracted from spectra in (D) and fig. S4. Gray curves in (D) and (E): Lindblad simulation results (see fig. S14, simulation parameters: $T_{1}^{(1)}=8 \mathrm{~ns}, \Omega^{(1)} /\left(2 \pi V_{\mathrm{RF} 1}\right)=0.16 \mathrm{MHz} / \mathrm{mV}, T_{1}^{(2)}=150 \mathrm{~ns}, \Omega^{(2)} /\left(2 \pi V_{\mathrm{RF} 2}\right)=$ $0.24 \mathrm{MHz} / \mathrm{mV}, 0.4<T<0.5 \mathrm{~K})$. 

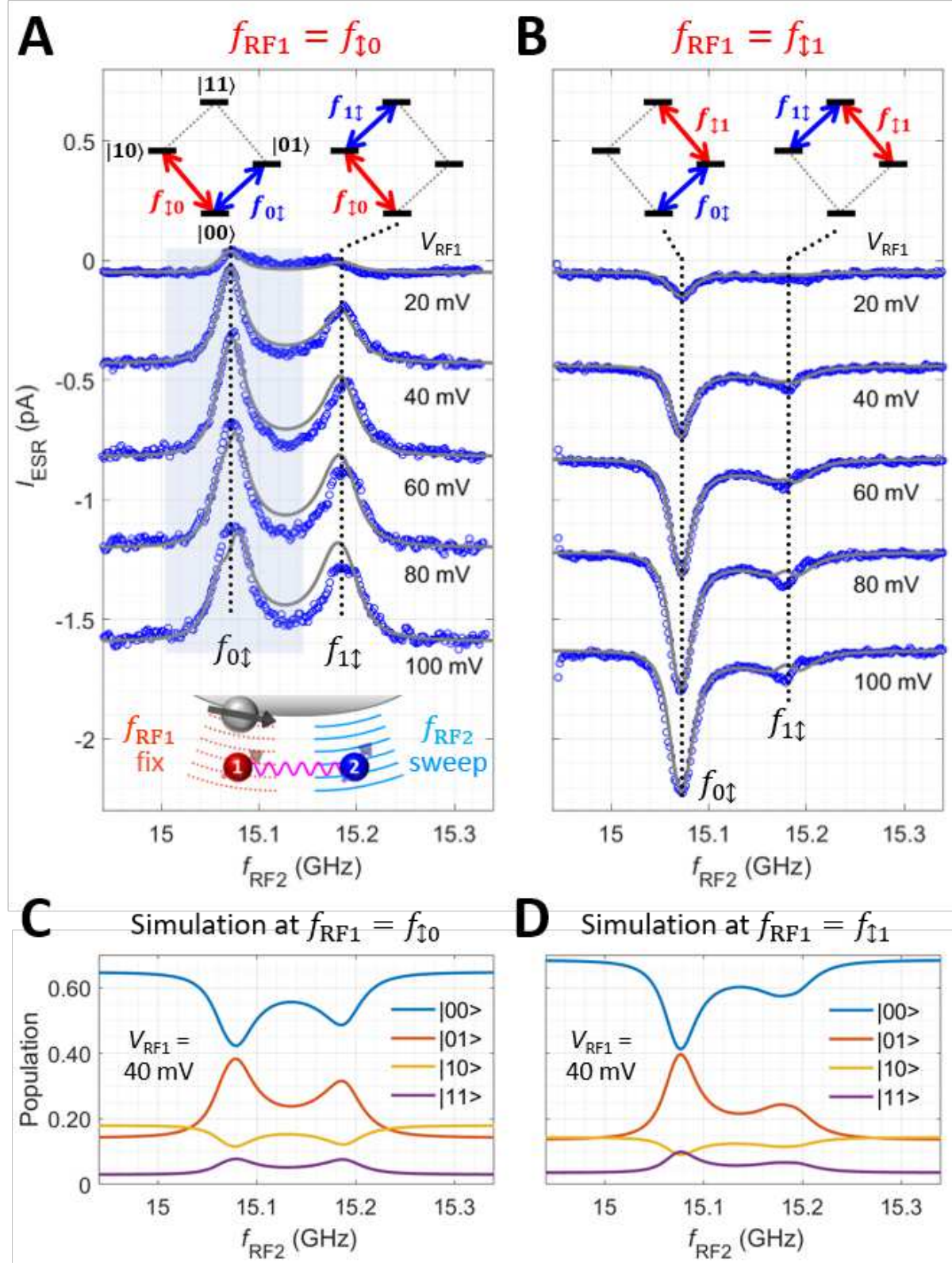

Fig. 2. Electron-electron double resonance spectroscopy. (A, B) Double resonance spectra with the tip positioned on Ti-1 at varying $V_{\mathrm{RF} 1}$, with $f_{\mathrm{RF} 1}$ fixed at $f_{\uparrow 0}=16.289 \mathrm{GHz}$ in (A) and $f_{\uparrow 1}=$ $16.401 \mathrm{GHz}$ in (B). Gray solid curves: Lindblad simulation results (19). Successive spectra are shifted by $-0.4 \mathrm{pA}$. Upper insets depict the ESR transitions involved at the peak frequencies. Lower inset in (A) shows the measurement scheme $\left(I_{\mathrm{DC}}=20 \mathrm{pA}, V_{\mathrm{DC}}=50 \mathrm{mV}, V_{\mathrm{RF} 2}=30 \mathrm{mV}, 0.4<\right.$ $T<0.5 \mathrm{~K}$ ). (C, D) Simulated populations of the four spin states at $V_{\mathrm{RF} 1}=40 \mathrm{mV}$. Simulation parameters same as Fig. 1D. 


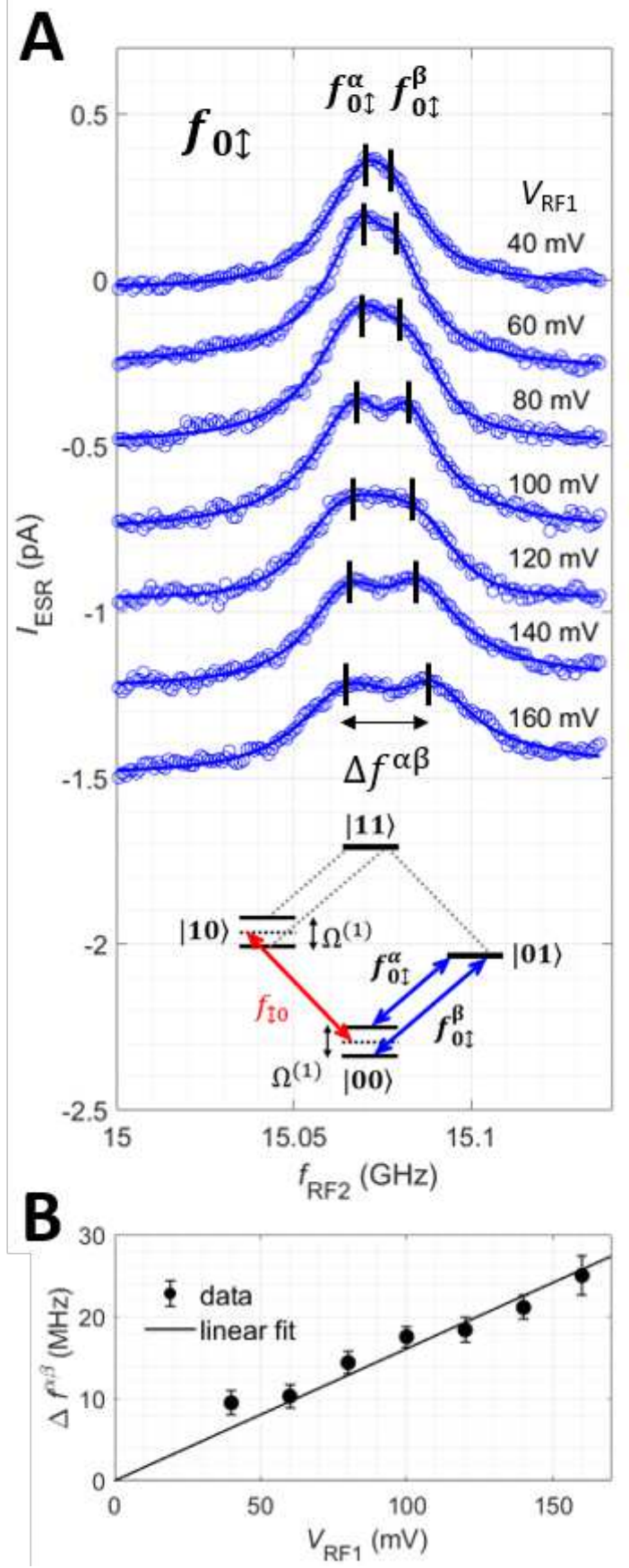

Fig. 3. AC Stark effect at strong driving of Ti-1. (A) Double resonance spectra of the Ti-2 resonance at $f_{0 \uparrow}$, showing that the peak splits as $V_{\mathrm{RF} 1}$ is increased. Inset: schematic illustrating the origin of the splitting according to the dressed-atom model (27). (B) Frequency difference between the two sub-peaks, $\Delta f^{\alpha \beta}=f_{1 \uparrow}^{\beta}-f_{1 \uparrow}^{\alpha}$ (fig. S7). A linear fit to $\Delta f^{\alpha \beta}\left(V_{\mathrm{RF} 1}\right)$ gives the Ti-1 Rabi rate $\Omega^{(1)} /\left(2 \pi V_{\mathrm{RF} 1}\right)=0.160 \pm 0.015 \mathrm{MHz} / \mathrm{mV}$. 

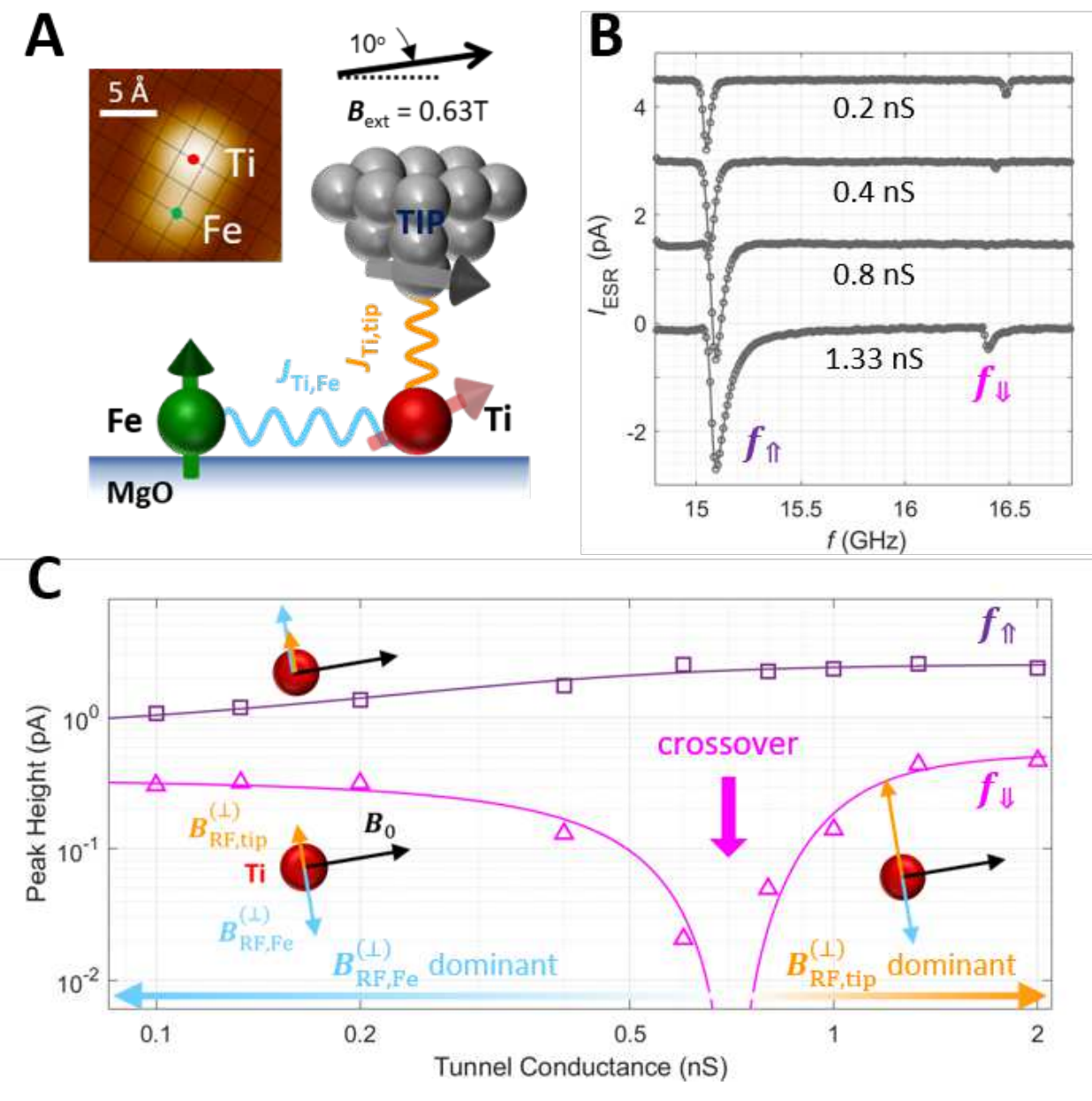

Fig. 4. ESR of Ti under the influence of a nearby Fe atom. (A) STM topographic image and schematic of magnetic interactions among Ti, Fe and tip. (B) ESR spectra measured on Ti at varying tunnel conductance. Successive spectra are shifted by $1.5 \mathrm{pA}\left(I_{\mathrm{DC}}=20 \mathrm{pA}, V_{\mathrm{DC}}=50 \mathrm{mV}\right.$, $V_{\mathrm{RF}}=30 \mathrm{mV}, T=0.7 \mathrm{~K}$ ). (C) ESR peak height at resonances $f_{\Uparrow}$ (purple) and $f_{\Downarrow}$ (magenta) as a function of tunnel conductance (data from (B) and fig. S18). Peak height at $f_{\Downarrow}$ showing a crossover near $0.7 \mathrm{nS}$, where the peak vanishes. The insets illustrate how the two transverse RF magnetic fields $\boldsymbol{B}_{\mathrm{RF}, \text { tip }}^{(\perp)}$ and $\boldsymbol{B}_{\mathrm{RF}, \mathrm{Fe}}^{(\perp)}$ add to (or cancel) each other. $\boldsymbol{B}_{0}$ is the static magnetic field. Solid curves are fits to the data using the model in Supplementary Text Section 6. 


\section{Supplementary Files}

This is a list of supplementary files associated with this preprint. Click to download.

- ELDORSI20210903.docx 